\title{
Parameters Designing of Slide Mode Variable Structure Controller of Bus Voltage of DC Microgrid Based on Proportion Switching Function
}

\author{
Zhenchuan Sun ${ }^{1,2}$, Jiasheng Zhang ${ }^{1}$ and Zhen Guo ${ }^{1,3}$ \\ ${ }^{1}$ College of Information and Control Engineering, China University of Petroleum(East China), Qingdao, Shandong, China \\ ${ }^{2}$ School of Mechanical and Electronic Engineering, Zaozhuang University, Zaozhuang, Shandong, China \\ ${ }^{3}$ College of Automation Engineering, Qingdao University of Technology, Qingdao, Shandong, China \\ Corresponding Email: author@e-mail.org
}

\begin{abstract}
Constant value control of the DC-bus voltage is a essential problem of the control system of the DC microgrids. DC-DC converters are applied in parallel to realize the transform of energy from the distributed generations (DGs) to the DC-bus. Droop control methods are applied to the DC-bus voltage while PI controllers are used in controlling the duty ratios of the converters. This method may bring out the slow response speed of the system accompanied by the large ripple of the voltage. The slide mode variable structure control can speed up the response and reduce the ripple of the voltage as well. In the traditional slide mode control based on the proportion switching function, the denominator of the transfer function of the controlled plant is a second-order characteristic polynomial without the constant term. The denominators of the transfer functions of the buck DC-DC converters contain the constant terms. The designing of the parameters of the slide mode control based on the proportion switching function is analyzed based on mathematics deductions. Simulation results show that the selected parameters can not only speed up the response of the system but also greatly reduce the ripple of the voltage.
\end{abstract}

\section{Introduction}

To solve the problem caused by energy voltage and envi-ronmental pollution, more and more attentions are payed to the distributed power generation technology. There are many advantages in the DGs such as lesspollutions, higher energy efficiency, and flexible loca-tions. Compared with the centralized power generations, it save the operating costs with reducing the line losses. With the development of the power electronics and themodern control theory, the microgrid emerges as anorganic whole combined with DGs, loads, energystorage devices and control devices. It may be operatedby the grid-connected mode and the island mode. ACmicrogrids are the main form of the microgrids.DC-buses are applied to transform the energy from theDGs to the loads in the DC microgrids. The reliabilityand controllability is improved as the control of thefrequency and the phase position is undesired in the DCmicrogrid [1,2].

Constant value control of the DC-bus voltage is aessential problem of the control system of the DC microgrid [3]. The droop control method is applied in the traditional control method of the dc-bus voltage [4]. The duty ratios of the DC-DC converters are controlled by the PI controllers which make the dc-bus voltage constant [5]. With the droop controllers and the PI controllers applied to the system, the response time may be long and the ripple of the dc-bus voltage may be large. In the dynamic process of the control system, the slide mode variable structure controller can vary on purpose according to the current state of the system which make the state move along the predetermined path of the slide state. The fast response may reduce the ripple of the dc-bus voltage and show the strong robustness [6].

By analyzing the parameters designing of the slide mode variable structure controller based on the proportion switching function, using the transform

function of the buck DC-DC converters with small-signal equivalent model, this paper makes a lot of theoretical calculations as the selecting of the parameters of the controller. With the phase plane as the analytical tool, the motion characteristics and stability is analyzed while the method proposed in this paper is tested by Matlab/Simulink. The simulation results show that the selected parameters can speed up the response and reduce the ripple of the dc-bus voltage. The robustness is present in the proposed method of the designing of the parameters. 


\section{The circuit of the DC microgrid control system}

The circuit of the DC microgrid control system is shown as Figure 1.

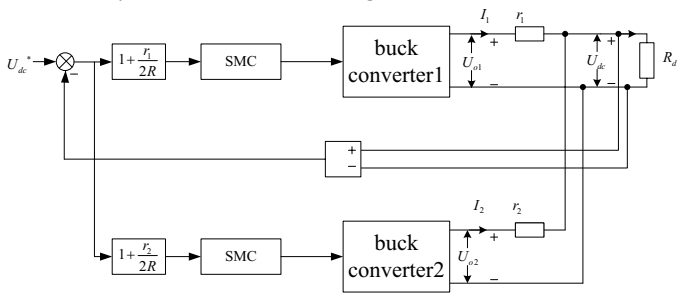

Fig. 1. The circuit of the DC microgrid control system.

In the DC microgrid control system, two buck DC-DC converters are applied to realize the transform of the energy. The values of the inductances, capacitances and resistances are the same while the input voltages of them are not, so as to simplify the analysis. The modified droop control strategy is used to realize the current-sharing control of the two converters $[7,8]$. The SMC is the slide mode variable structure controller based on the proportion switching function.The transfer function of the buck converter is approximate using small-signal equivalent model. The impedances of the cables from the convertes to the load is supposed as the pure resistances. The load is a equivalent pure resistor load $[9,10]$.

\section{Parameters designing of the SMC of the DC microgrid system}

The transfer function of the buck converter of the small-signal equivalent model is as formula (1).

$$
\left.G(s)\right|_{U_{i}=\text { const }}=\frac{U_{o}(s)}{d(s)}=\frac{U_{i}}{L C s^{2}+\frac{L}{R} s+1}=\frac{\frac{U_{i}}{L C}}{s^{2}+\frac{1}{R C} s+\frac{1}{L C}}
$$

While $U_{i}$ is the input voltage of the buck converter. It is not the same as the transfer function of the SMC based on the proportion switching function in form because of its denominator is a second-order characteristics polynomial with a constant term. The form of common transfer function is $\frac{b}{s^{2}+a s}$.

Suppose that $G(s)=\frac{U_{o}(s)}{d(s)}=\frac{x_{1}(s)}{u(s)}$, formula (1) can be rewrited as the state space model as formula (2).

$$
\begin{gathered}
\dot{x}=A x+B u \\
{\left[\begin{array}{c}
\dot{x}_{1} \\
\dot{x}_{2}
\end{array}\right]=\left[\begin{array}{cc}
0 & 1 \\
-\frac{1}{L C} & -\frac{1}{R C}
\end{array}\right]\left[\begin{array}{l}
x_{1} \\
x_{2}
\end{array}\right]+\left[\begin{array}{c}
0 \\
\frac{U_{i}}{L C}
\end{array}\right] u}
\end{gathered}
$$

While $\dot{x}=\left[\begin{array}{c}\dot{x}_{1} \\ \dot{x}_{1}\end{array}\right]$.

According to the control method of the SMC based on the propotion swithching function, the position command signals of the two SMC controllers are the output voltages of the two converters. That is to say

$$
\left\{\begin{array}{c}
e=r-x_{1}=U_{\text {oref }}-x_{1} \\
\dot{e}=\dot{r}-\dot{x}_{1}=-x_{2}
\end{array}\right.
$$

From formula (2) and (3),we can get

$$
-\left[\begin{array}{c}
\dot{e} \\
\ddot{e}
\end{array}\right]=\left[\begin{array}{cc}
0 & 1 \\
-\frac{1}{L C} & -\frac{1}{R C}
\end{array}\right]\left[\begin{array}{c}
U_{\text {oref }}-e \\
-\dot{e}
\end{array}\right]+\left[\begin{array}{c}
0 \\
\frac{U_{i}}{L C}
\end{array}\right] u
$$

While $u=(\alpha|e|+\beta|\dot{e}|) \operatorname{sgn}(s), \quad s=c e+\dot{e}$. This paper focuses on the designing of the parameters $\alpha, \beta$ and $c$.

In order to simplify the problem, we make following assumptions:

(1) We suppose that $\alpha>0, \beta>0$, and $c<0$. 
(2) Only the locus of the slide mode from the initial point to the switching surface is considered

(3) The two SMCs of the two converters are designed on the same theory.

That is to say, the only situation that should be researched is that $e>0, \dot{e}<0$ and $s>0$. This situation shows that the actual value is gradually approaching the setting value.

If $s>0$,then $\operatorname{sgn}(s)=1, u=\alpha e-\beta \dot{e}$.

$$
\begin{array}{r}
-\left[\begin{array}{l}
\dot{e} \\
\ddot{e}
\end{array}\right]=\left[\begin{array}{cc}
0 & 1 \\
-\frac{1}{L C} & -\frac{1}{R C}
\end{array}\right]\left[\begin{array}{c}
U_{\text {oref }}-e \\
-\dot{e}
\end{array}\right]+\left[\begin{array}{c}
0 \\
\frac{U_{i}}{L C}
\end{array}\right]\left[\begin{array}{ll}
\alpha & -\beta
\end{array}\right]\left[\begin{array}{l}
e \\
\dot{e}
\end{array}\right] \\
{\left[\begin{array}{c}
\dot{e} \\
\ddot{e}
\end{array}\right]=\left[\begin{array}{cc}
0 & 1 \\
-\frac{1+U_{i} \alpha}{L C} & \frac{U_{i} \beta}{L C}-\frac{1}{R C}
\end{array}\right]\left[\begin{array}{l}
e \\
\dot{e}
\end{array}\right]+\left[\begin{array}{c}
0 \\
\frac{U_{\text {oref }}}{L C}
\end{array}\right]}
\end{array}
$$

According to the reaching condition of the slide mode, we get

$$
s \dot{s}<0
$$

Using formula (6) we can get

$$
\text { So, } \dot{s}<0 \text {. }
$$

$$
\begin{aligned}
& \dot{s}=c \dot{e}+\ddot{e}=\left[\begin{array}{ll}
c & 1
\end{array}\right]\left[\begin{array}{l}
\dot{e} \\
\ddot{e}
\end{array}\right] \\
& =\left[\begin{array}{ll}
c & 1
\end{array}\right]\left\{\left[\begin{array}{cc}
0 & 1 \\
-\frac{1+U_{i} \alpha}{L C} & \frac{U_{i} \beta}{L C}-\frac{1}{R C}
\end{array}\right]\left[\begin{array}{l}
e \\
\dot{e}
\end{array}\right]+\left[\begin{array}{c}
0 \\
\frac{U_{\text {oref }}}{L C}
\end{array}\right]\right\} \\
& =\frac{U_{\text {oref }}}{L C}-\frac{1+U_{i} \alpha}{L C} e+\left(\frac{U_{i} \beta}{L C}+c-\frac{1}{R C}\right) \dot{e} \\
& =\frac{U_{\text {oref }}}{L C}-\frac{1+U_{i} \alpha}{L C} e+\frac{U_{i} \beta R+R L C c-L}{R L C} \dot{e}<0
\end{aligned}
$$

If $\frac{U_{i} \beta R+R L C c-L}{R L C}>0$, then the last line of formula (8) can be calculated as

$$
\dot{e}<\frac{\left(1+U_{i} \alpha\right) R}{U_{i} \beta R+R L C c-L} e-\frac{R U_{\text {oref }}}{U_{i} \beta R+R L C c-L}
$$

Since $1+U_{i} \alpha>0$, so formula (9) can be expressed as the straight line $l_{2}$ in Figure 2 which phase plane plot with $e$ as its $\mathrm{x}$ axes and $\dot{e}$ as its y axes.

As shown in Figure 2, the x-coordinate of $A$ is $\frac{U_{\text {oref }}}{1+U_{i} \alpha}$, while the y-coordinate of $B$ is $-\frac{R U_{\text {oref }}}{U_{i} \beta R+R L C c-L}$.

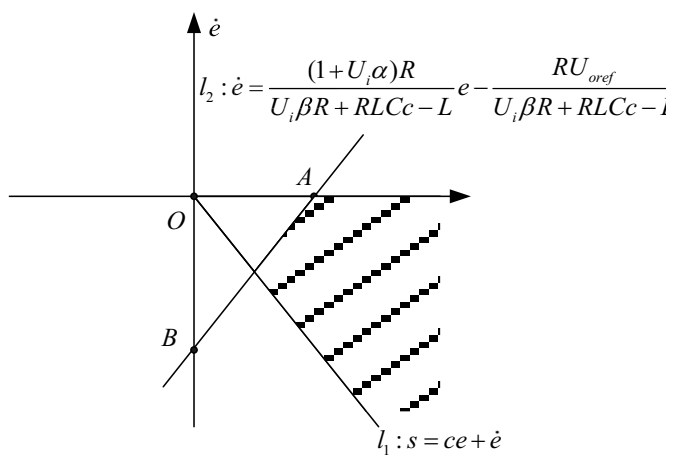

Fig. 2. The phase plane plot of the SMC. 
According to the plane geometry,inequality systems

$$
\left\{\begin{array}{c}
s=c e+\dot{e}>0 \\
\dot{s}=\frac{U_{\text {oref }}}{L C}-\frac{1+U_{i} \alpha}{L C} e+\frac{U_{i} \beta R+R L C c-L}{R L C} \dot{e}<0
\end{array}\right.
$$

is expressed by the shaded area in Figure 2.That is to say, the locus of the slide mode from the initial point to the line $l_{1}$ should be within the shaded area in Figure 2.

So,we can get inequality constrains as follows:

Formula (11) can be calculated as

$$
\left\{\begin{array}{c}
\frac{U_{\text {oref }}}{1+U_{i} a}<U_{\text {oref }} \\
U_{i} \beta R+R L C c-L>0
\end{array}\right.
$$

$$
\left\{\begin{array}{c}
\alpha>0 \\
\beta>\frac{L-R L C c}{U_{i} R} \\
\frac{L-U_{i} \beta R}{R L C}<c<0
\end{array}\right.
$$

If $\frac{U_{i} \beta R+R L C c-L}{R L C}<0$, then the last line of formula (8) can be calculated as

$$
\dot{e}>\frac{\left(1+U_{i} \alpha\right) R}{U_{i} \beta R+R L C c-L} e-\frac{R U_{\text {oref }}}{U_{i} \beta R+R L C c-L}
$$

As the analysis mentioned above, formula (13) can be expressed as the straight line $l_{3}$ in Figure 3.

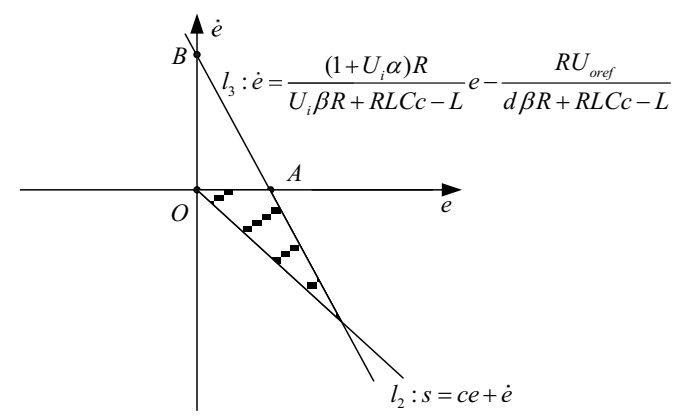

Fig. 3. The phase plane plot of the SMC.

Suppose the locus of the slide mode lies in the shaded area, we can get

$$
\frac{U_{\text {oref }}}{1+U_{i} a}>U_{\text {oref }}, 0<1+U_{i} \alpha<1
$$

Formula (14) is in conflict with the initial conditions including that $\alpha>0$. So the suppose that $\frac{U_{i} \beta R+R L C c-L}{R L C}>0$ is false.

From the above, the parameters should satisfy the following inequality constrains from the initial point to the switching surface is formula (12).

\section{Simulation Results and Analysis}

The circuit parameters of the two buck converters are as follows: $R=0.6 \Omega, L=6 \times 10^{-5} \mathrm{H}, C=4 \times 10^{-3} \mathrm{~F}$, $f_{s}=4 \times 10^{4} \mathrm{~Hz}$. The input voltages of the two buck converters are $U_{i 1}$ and $U_{i 2} . U_{i 1}=200 \mathrm{~V}$, 
$U_{i 2}=400 \mathrm{~V}$. The initial duty cycles of the two buck converters are $d_{1}$ and $d_{2} . d_{1}=56.25 \%$,

$d_{2}=31.25 \%$. The reference of the dc-bus voltage changes with time as follows:

$$
\begin{gathered}
t: \quad 0 \sim 0.1 s, U_{\text {dcref }}=100 \mathrm{~V} \\
0.1 s \sim 0.2 s, U_{\text {dcref }}=120 \mathrm{~V} \\
0.2 \sim 0.3 s, U_{\text {dcref }}=110 \mathrm{~V} \\
0.3 \sim 0.4 s, U_{\text {dcref }}=130 \mathrm{~V}
\end{gathered}
$$

The parameters $\alpha, \beta$ and $c$ are selected by the formula (12) are as follows:

$$
\left\{\begin{array}{c}
\alpha>0 \\
\beta>\frac{6 \times 10^{-5}-1.44 \times 10^{-7} c}{1.2 \times 10^{2}}>\frac{6 \times 10^{-5}-1.44 \times 10^{-7} c}{2.4 \times 10^{2}} \\
\frac{6 \times 10^{-5}-2.4 \times 10^{2} \beta}{1.44 \times 10^{-7}}<\frac{6 \times 10^{-5}-1.2 \times 10^{2} \beta}{1.44 \times 10^{-7}}<c<0
\end{array}\right.
$$

As the formula (15) mentioned above, we take $\alpha=500, \beta=10, c=-50$ as an example.

The simulation results based on the theory above are figures as follows:

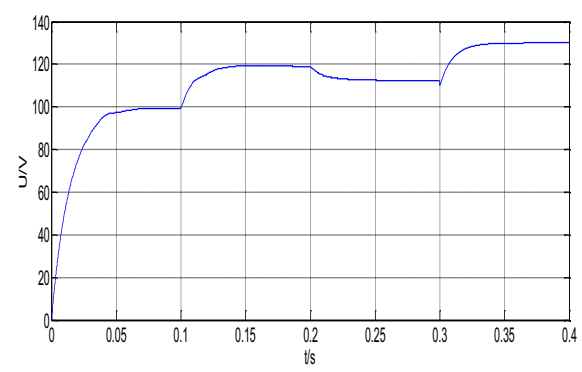

Fig. 4. The dc-bus voltage of the DC microgrid based on SMC.

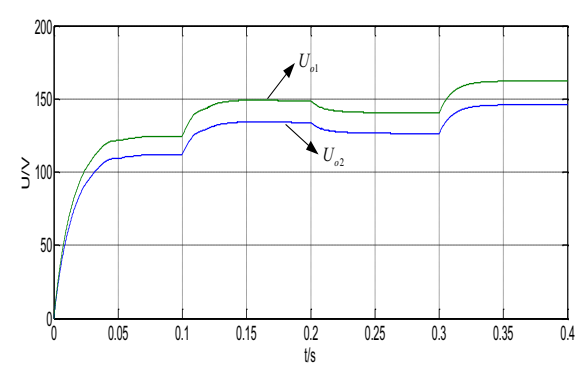

Fig. 5. The output voltages of the two buck converters based on SMC. 


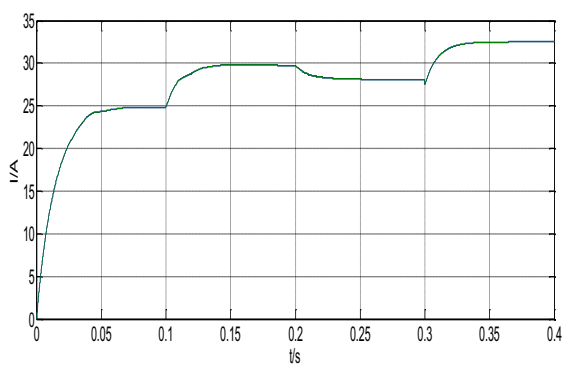

Fig. 6. The output currents of the two buck converters based on SMC.

By constract, the simulation results based on the PI controllers are shown as follows. The parameters of the two PI controllers are $k_{P_{1}}=1.25, k_{I_{1}}=1 ; k_{P_{1}}=1.5, k_{I_{1}}=1$ [11]. Based on that the response time of PI controllers is longer than it of SMC.The reference of the dc-bus voltage is reset as follows:

$$
\begin{gathered}
t: 0 \sim 5 s, U_{\text {dcref }}=100 \mathrm{~V}, \\
5 s \sim 10 s, U_{\text {dcref }}=120 \mathrm{~V}, \\
10 s \sim 15 s, U_{\text {dcref }}=110 \mathrm{~V}, \\
10 s \sim 15 s, U_{\text {dcref }}=130 \mathrm{~V} .
\end{gathered}
$$

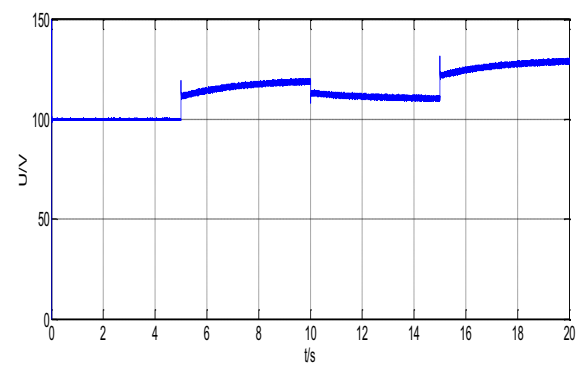

Fig. 7. The dc-bus voltage of the DC microgrid based on PI controller.

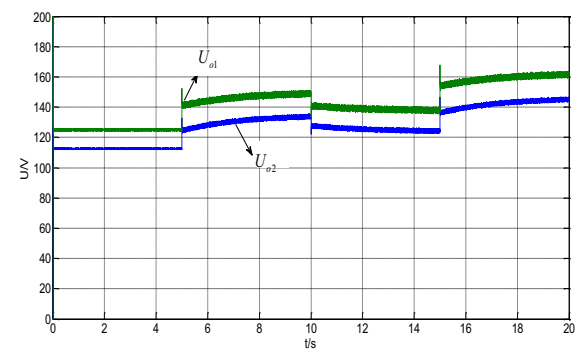

Fig. 8. The out voltages of the two buck converters based on PI controller. 


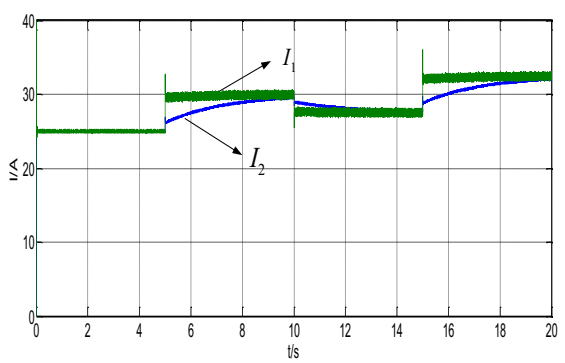

Fig. 9. The output currents of the two buck converters based on PI controller.

From Figure 4 to Figure 6 we can come to the conclusion that the actual dc-bus can track its four different set values within $0.1 \mathrm{~s}$ based on the SMC.The output currents of the two buck dc-dc converters are identical.

From Figure 7 to Figure 9 we can come to the conclusion that the actual dc-bus can also track its four different set values based on the PI controllers. But the response time is $5 \mathrm{~s}$. The output currents of the two buck dc-dc converters are the same after $5 \mathrm{~s}$. Besides, from Figure 4 to Figure 9, we can get that the ripples of the voltages and the currents based on the PI controllers are larger than them based on the SMC.

The simulation results show that the SMC including reasonable parameters is superior to the PI control. The method of the parameter designing may also be applied to other controlled plant which transfer function has the second-order characteristic polynomial as its denominator including constant term.

\section{Conclusion}

Based on the mathematical derivations of the theory of the proportion switching function of the SMC, a new parameter designing of the SMC has been present in this paper. Taking the buck dc-dc converters of the DC microgrids as an example, using the transfer function between and duty cycle and the output voltage based on the small-signal model, this paper has provided us many valuable conclusions. The simulation results show that the SMC including the reasonable parameters is superior to the PI control. Furthermore, the research of the state which has reached the switching surface along with the change of the parameters of the SMC based on the proportion switching function will be dealt with in future.

\section{Acknowledgement}

First of all, I would like to extend my sincere gratitude to my supervisor, Zhang Jiasheng, for his instructive advice and useful suggestions on my thesis. I am deeply grateful of his help in the completion of this thesis. I am also deeply indebted to all the other tutors and teachers for their direct and indirect help to me. Finally, I am indebted to my mother and my wife for their continuous support and encouragement.

\section{References}

1. J.H.Zhang, W.Huang. Operation control and protection technology of Microgrid. 1-5(2009)

2. C.S.Wang. Analysis and simulation theory of Microgrid. Science Press, Beijing(2013)

3. W.M.Wu,Y.B.He,P.Geng. Transactions of China Electrotechnical Society, Vol.27,No.1, pp. 98-113, (2012)

4. F.Gao, Y.Gu, S.Bozhko, G.Asher, P. Wheeler.2014 $16^{\text {th }}$ European Conference on Power Electronics and Applications(2014)

5. D.H.Xu. Modeling and control of power electronics system. 43(2005)

6. J.K.Liu. MATLAB simulation for sliding mode control. 25-30(2005)

7. P. Chaudhari, M. Khadse, V. Jadhav, P. Patil, K. Daware, M.Rajwade, S.Sharma.2015 IEEE International Conference on Signal Processing,Informatics, Communication and Energy Systems,(2015)

8. L. Zhang, T.J. Wu,Y. Xing,K. Sun,J.M. Gurrero. IEEE Applied Power Electronics Conference and Exposition,1926-1932(2011)

9. S.Augustine ,M. K. Mishra, N.Lakshminarasamma. IEEE Transactions on Sustainable Energy. Vol.6,No.1, pp. 132-141(2015)

10. L. Zhang, T.J. Wu, Y. Xing, K. Sun, J.M. Gurrero. IEEE Applied Power Electronics Conference and Exposition, 1926-1932(2011)

11. J.K. Liu. MATLAB simulation for advanced PID control( $3^{\text {rd }}$ edition $), 1-8(2011)$ 